\title{
Association of 24-Hour Heart Rate Variability and Daytime Physical Activity: ALLSTAR Big Data Analysis
}

\author{
Junichiro Hayano*, Yuki Furukawa, Emi Yuda, Yutaka Yoshida \\ Nagoya City University Graduate School of Medical Science, 1 Kawasumi, Mizuho-cho Mizuho-ku, Nagoya \\ 467-8602, Japan.
}

* Corresponding author. Tel.: +81-52-853-8501; email: Hayano@med.nagoya-cu.ac.jp

Manuscript submitted June 4, 2017; accepted September 3, 2017.

doi: 10.17706/ijbbb.2018.8.1.61-67

\begin{abstract}
This paper reports the temporal structures of the relationships between 24-hour heart rate variability (HRV) and physical activity (PA) in clinical big data of simultaneously recorded electrocardiograms (ECG) and actigraphic data in 21,682 male and 29,446 female patients (age, 20-100 yr). We found that SDNN (standard deviation of 24-hour normal-to-normal R-R interval [NN interval] of ECG) and PA during daytime, evening, and nighttime decreased with age. In regression models adjusted for the effect of age, daytime PA explained $7 \%$ of inter-individual variance in SDNN, while evening and early morning PA explained only $<0.6 \%$ and nighttime PA negatively contributed to SDNN. Daytime but not nighttime PA is positively associated with increased 24-hour HRV.
\end{abstract}

Key words: Actigraphic data, biomedical big data, electrocardiogram, heart rate variability, lifestyle modification, physical activity.

\section{Introduction}

Increase in physical activity (PA) is an important life style modification for preventing cardiovascular diseases [1], [2]. Studies have reported that PA and exercise training prevent atherosclerotic coronary artery diseases and reduce coronary risk factors including elevated triglycerides, low high-density-lipoprotein (HDL) cholesterol, hypertension, glucose intolerance, and obesity [2]. On the other hand, PA is known to suppress heart rate variability (HRV) [3], [4] whose decreased during 24 hours is known to predict increased mortality risk among cardiovascular patients [5], [6]. Thus, it is unclear whether increased PA in daily life is associated positively with 24-hour HRV or rather negatively with it.

In the present study, we aimed at examining the relationship between HRV and PA during daily life. For this purpose, we used a biomedical database of clinical Holter monitoring that has been constructed by the Allostatic State Mapping by Ambulatory ECG Repository (ALLSTAR) project [7], [8]. The ALLSTAR project has started to construct a database of clinical 24-hour ambulatory electrocardiogram (ECG) that were recorded all over Japan and were referred for analysis to three ECG analysis centers (Suzuken Co., Ltd.) located in Tokyo, Nagoya, and Sapporo in Japan. By March 2016, >400 thousand anonymized ECG data have been collected and $>50$ thousand data are being added per year. After December 2010, actigraphic data obtained by Holter recorders with built-in three-axis accelerometers have also been added. In this study, we used simultaneously recorded ambulatory ECG and actigraphic data and analyzed the temporal structures of the relationships between 24-hour HRV and PA in different time zones of the day. 


\section{Methods}

The protocol of the present study has been approved by the Institutional Review Board of Nagoya City University Graduate School of Medical Sciences and Nagoya City University Hospital (No. 709). According to the Ethical Guidelines for Medical and Health Research Involving Human Subjects (by the Japanese Ministry of Education, Culture, Sports, Science and Technology and the Japanese Ministry of Health, Labour and Welfare, December 22, 2014, partly revised on February 28, 2017), the purposes and information utilized in the research projects have been public through the homepages of the Suzuken ECG analysis centers (http://www.suzuken.co.jp/product/holter/detail/) and that of the ALLSTAR research project (http://www.med.nagoya-cu.ac.jp/mededu.dir/allstar/), in which opportunities to refuse the uses of information are ensured for the research subjects.

\subsection{Database}

The 24-h ECG data were digitized at $125 \mathrm{~Hz}$ and were analyzed with Holter ECG analyzers (Cardy Analyzer 05, Suzuken Co., Ltd., Nagoya, Japan) by skilled medical technologists at the three ECG analysis centers. The temporal positions of all R-waves were detected, the rhythm annotations were given to all QRS complexes, and all errors in automated analysis were corrected manually by the technologists. The suspicious outcomes of the analysis (approximately 40\% of data) have been reviewed by contracted cardiologists. After these analyses, the following information for the entire recording length (usually for 24 h) have been stored in the database: (1) times of all $R$ waves after the start of recoding in ms, (2) R-R intervals in ms, (3) rhythm annotation labels of all QRS complexes, and (4) ST levels following the QRS complexes for channels 1 and 2 .

For the 24-h actigraphic data, three-axis accelerograms were digitized at $31.25 \mathrm{~Hz} / \mathrm{channel}$. The data were stored in the database for the entire recording length of $\mathrm{x}^{-}, \mathrm{y}^{-}$, and $\mathrm{z}$-axis data corresponding to the mediolateral, longitudinal, and anteroposterior accelerations, respectively.

For the present study, we extracted data from the ALLSTAR database with the following inclusion criteria: (1) data from 24-hour ECG and three-axis accelerograms that were recorded simultaneously by specific models of Holter recorders with built-in three-axis accelerometers (Cady 303 pico or pico+, Suzuken Co., Ltd., Nagoya, Japan), (2) data from subjects aged 20-100 years, and (3) R-R interval time series in which $\geq$ $80 \%$ of recorded heartbeats were in sinus rhythm for all time zones of 9-17 h, 17-24 h, 0-6 h, and 6-9 h.

\subsection{Data Analysis}

According to the guideline for HRV analysis [9], only R-R interval data consisting of consecutive sinus beats (NN interval) were extracted and their standard deviation of NN interval over 24 hours (SDNN) was calculated as an index of 24-hour HRV. Using only NN intervals was to avoid the effects of pathological arrhythmias on HRV measurements. Then, using the time of each interval datum, the time series data of NN interval were classified into 4 groups depending on the time of occurrence; i.e., daytime (DT, 09:00-17:00), evening (EV, 17:00-24:00), nighttime (NT, 00:00-06:00), and early morning (EM, 06:00-09:00), and averaged over each time zone as mean $N$ interval ( $\mathrm{MNN}_{\mathrm{DT}}, \mathrm{MNN}_{\mathrm{EV}}, \mathrm{MNN}_{\mathrm{NT}}$, and $\left.\mathrm{MNN}_{\mathrm{EM}}\right)$.

For actigraphic data, the time series data of acceleration signals in $\mathrm{x}, \mathrm{y}$, and $\mathrm{z}$ axes were bandpass filtered (0.02-0.08 Hz) to remove baseline trend and high-frequency noise, and were composed into the magnitude of $\mathrm{PA}$, with the following equation.

$$
P A(t)=\sqrt{x(t)^{2}+y(t)^{2}+z(t)^{2}}
$$

Then, the data points of PA(t) were grouped into the same time zones as those for NN intervals and averaged over each zone as $\left(\mathrm{PA}_{\mathrm{DT}}, \mathrm{PA}_{\mathrm{EV}}, \mathrm{PA}_{\mathrm{NT}}\right.$, and $\left.\mathrm{PA} \mathrm{E}_{\mathrm{EM}}\right)$. 


\subsection{Statistical Analysis}

We used program package of Statistical Analysis System (SAS institute, Cary, NC, USA) for statistical analyses. We evaluated the effects of age, sex, and MNN and PA at different time zones on SDNN with the General Linear Model procedure and the inter-individual variances of SDNN explained by PA with the Regression procedure.
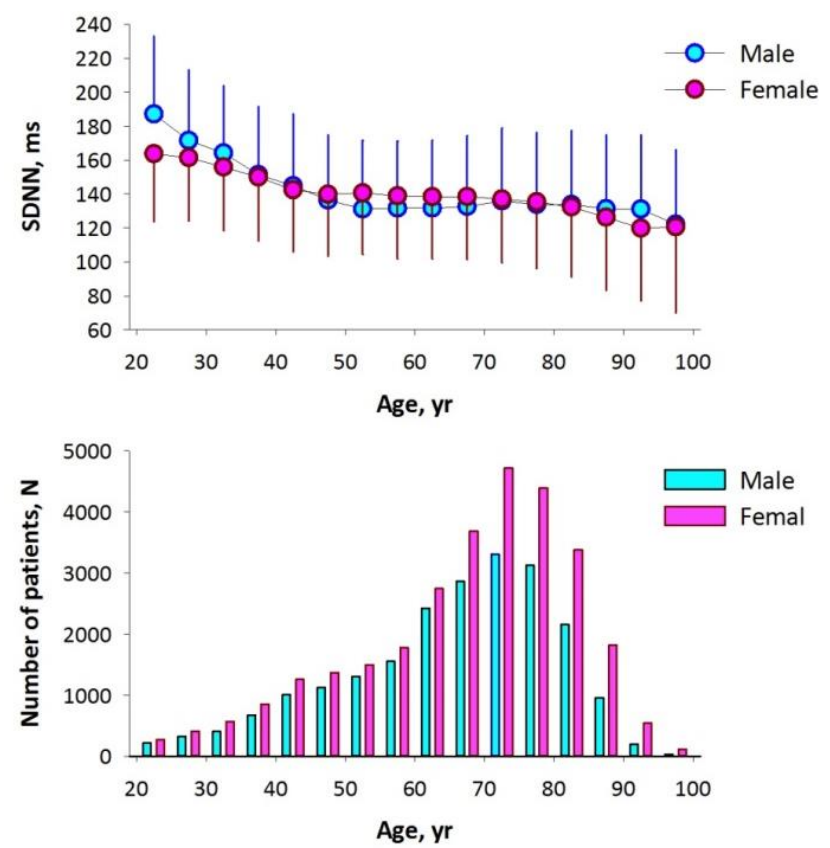

Fig. 1. SDNN (standard deviation of normal-to-normal R-R interval for 24 hour) in patients divided by age and sex (upper panel) and the number of patients in each age group and sex (lower panel). Error bars in upper panel denote SD.
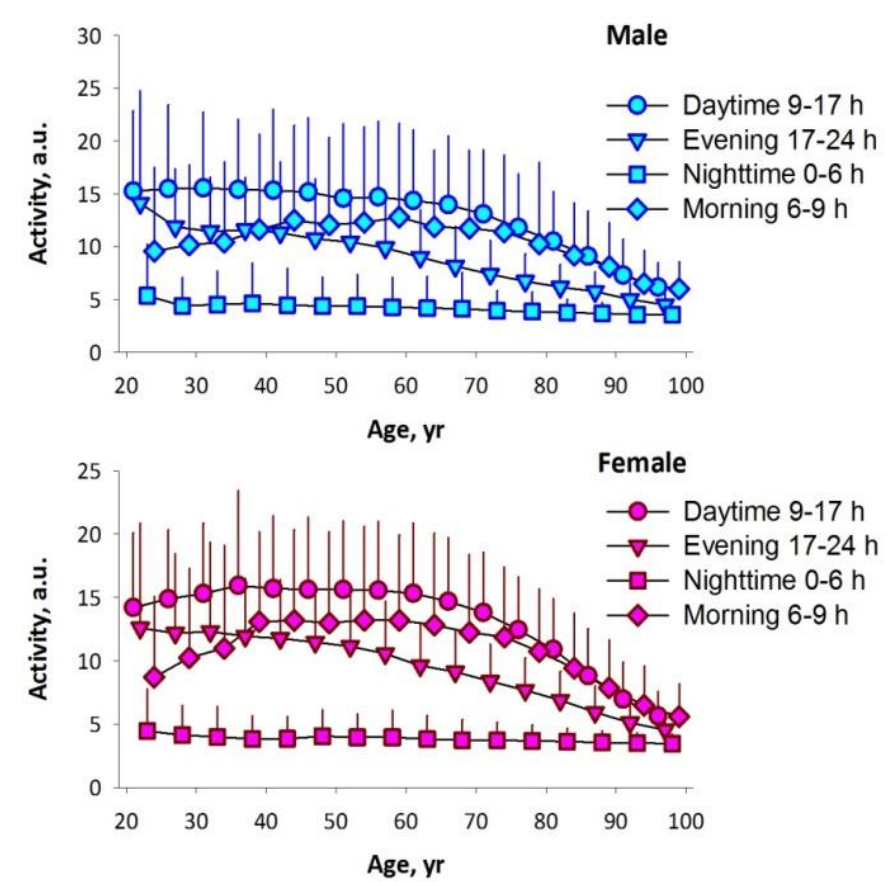

Fig. 2. Physical activity in different time zone of the day in male (upper panel) and female (lower panel) patients divided by age. Error bars denote SD. The number of patients for each age group and sex is shown in the lower panel of Fig. 1. 
Table 1. Regression Models of SDNN by Age and PA in Different Time Zones of the Day

\begin{tabular}{ccccc}
\hline & Explained variance, $\%$ & Regression coefficient (SE) & F (5,1) & P \\
\hline Male & & & & \\
Age & 0.57 & $-0.23(0.02)$ & 125.8 & $<.0001$ \\
PADT $_{\text {PAEV }}$ & 7.63 & $1.49(0.05)$ & 861.7 & $<.0001$ \\
PANT $_{\text {PAEM }}$ & 0 & $0.05(0.07)$ & 0.5 & 0.4 \\
& 0.24 & $-0.82(0.11)$ & 57.8 & $<.0001$ \\
Female & 0.37 & $0.43(0.04)$ & 108.9 & $<.0001$ \\
Age & & & & \\
PADT & & & 191.7 & $<.0001$ \\
PAEV & 0.51 & $-0.22(0.02)$ & 1057.9 & $<.0001$ \\
PANT $_{\text {PAEM }}$ & 7.43 & $1.64(0.05)$ & 14.7 & 0.0001 \\
\hline
\end{tabular}

$\mathrm{PA}_{\mathrm{DT}}, \mathrm{PA}_{\mathrm{EV}}, \mathrm{PA}_{\mathrm{NT}}$, and $\mathrm{PA} \mathrm{EM}_{\mathrm{EM}}$ indicate physical activity in daytime (09:00-17:00), evening (17:00-24:00), nighttime (00:00-06:00), and early morning (06:00-09:00), respectively.

Table 2. Partial Correlation of SDNN with Mean N-N Intervals (MNN) and PA in Different Time Zones (the Effect of Age Is Partialed Out)

\begin{tabular}{lcc}
\hline & Male & Female \\
\hline MNN 24-hour & $\mathrm{N}=21682$ & $\mathrm{~N}=29446$ \\
MNN $_{\mathrm{DT}}$ & 0.50 & 0.50 \\
MNNEV $_{\text {MNN }}$ & 0.31 & 0.29 \\
MNNEM $_{\text {MNN }}$ & 0.50 & 0.50 \\
$\Delta$ MNN $\left._{\mathrm{NT}}-\mathrm{MNN}_{\mathrm{DT}}\right)$ & 0.72 & 0.74 \\
\hline
\end{tabular}

$P<0.0001$ for all correlation coefficients.

$\mathrm{MNN}_{\mathrm{DT}}, \mathrm{MNN}_{\mathrm{EV}}, \mathrm{MNN}_{\mathrm{NT}}$, and $\mathrm{MNN}_{\mathrm{EM}}$ indicate mean $\mathrm{NN}$ intervals in daytime (09:00-17:00), evening (17:00-24:00), nighttime (00:00-06:00), and early morning (06:00-09:00), respectively.

\section{Results}

HRV and PA data meeting with the inclusion criteria were obtained from 21,682 male (age \pm SD, $65 \pm 15$ year) and 29,446 female (66 \pm 15 year) patients. All data have been recorded between December 2010 and March 2016.

Fig. 1 shows the distribution of SDNN and the number of patients in each age group and sex. In both sexes, SDNN decreased with age $(P<0.0001)$ and was greater in female than male after adjusting the effect of age $(P<0.0001)$.

Fig. 2 shows the distribution of PA at different time zones in patients in each age group and sex. In both sexes, $\mathrm{PA}_{\mathrm{DT}}, \mathrm{PA}_{\mathrm{EV}}$, and $\mathrm{PA}_{\mathrm{NT}}$ decreased with age (for all, $P<0.0001$ ), while $\mathrm{PA}_{\mathrm{EM}}$ showed a parabolic-curve relationships with age. After adjusting for the effect of age, PA was greater in female than male in all time zones but $\mathrm{PA} \mathrm{EM}_{\mathrm{EM}}$ which was greater in males (for all, $P<0.0001$ ).

Table 1 shows the regression models of SDNN by age and PA at different time zones of the day. The model revealed that after adjusting for the effect of age, $\mathrm{PA}_{\mathrm{DT}}$ is associated positively with SDNN and it explains $7.63 \%$ in male and $7.43 \%$ in female of inter-individual variances in SDNN. On the other hand, $P A_{E V}$ and PAEM showed no substantial effects in either sex (explained variances, 0-0.41\%). Furthermore, $\mathrm{PA}_{\mathrm{NT}}$ showed 
negative effect on SDNN in both sexes.

To clarify the possible reasons for that only PADT is substantially associated with SDNN, the relationships between SDNN and MNN in different time zones. As shown in Table 2, SDNN was correlated positively with $\mathrm{MNN}$ in all time zones and the difference between nighttime and daytime ( $\mathrm{MNN}_{\mathrm{NT}}-\mathrm{MNN}_{\mathrm{DT}}$ ), it correlated most strongly with $\mathrm{MNN}_{\mathrm{NT}}$ and most weakly with $\mathrm{MNN}_{\mathrm{DT}}$ in both sexes.

\section{Discussions}

In this study, we analyzed the temporal structure of the relationships between SDNN and PA in different time zone of the day in the ALLSTAR database of ECG and actigraphic data obtained from adult patients who underwent clinical 24-hour Holter monitoring. We found that only daytime PA had a substantial positive effect on SDNN while nighttime PA had a negative effect on it. Our findings support for the hypothesis that daytime PA is associated with increased 24-hour HRV.

There are many studies reporting increases in HRV with exercise training [10]-[12]. Those studies suggest that exercise training improves HRV in a variety of clinical conditions including post-myocardial infarction, chronic heart failure, unstable angina, and diabetes mellitus. Although such evidence suggests that the greater PA during daily life is associated with the increased HRV, there are few studies that have directly observed the relationships.

In the present study, we utilized the ALLSTAR database of Holter ambulatory ECG monitoring for analyzing the association between PA and HRV in daily life. Fortunately, a substantial part of ambulatory ECG data stored in this database has been recorded with specific models of Holter ECG recorders with a standardized built-in three-axis accelerometer. Taking advantage of this merit, we were able to estimate and compared the PA with the same uniform measure of PA in >50 thousand of patients.

Because of the observational nature of this study, we cannot determine the exact mechanisms for the association between daytime PA and 24-hr HRV. Some speculations, however, may be possible from the correlations between SDNN and MNN during different time zones. We observed that SDNN is correlated strongly with nighttime MNN but weakly with daytime MNN. Thus, even if a high level of daytime PA may reduce daytime MNN through cardiac vagal suppression and/or sympathetic activation, it would have a small effect on SDNN. Furthermore, because a resting bradycardia is a well-stablished consequence of exercise training [10], [13], daytime PA may increase nighttime MNN. Our observation that nighttime PA was negatively associated with SDNN may also support for the importance of nighttime MNN for maintaining a high level of SDNN. From these, we may suggest the hypothesis that daytime PA is associated with higher SDNN through higher cardiac vagal activity during nighttime.

This study has several limitations. First, because this is an observational study, we cannot determine the causal relationships between daytime PA and HRV or exclude the possibility that the apparent association between them is mediated by a third factor. Second, because we used the database of clinical data, the observations may be biased by the type and severity of disease among patients. The database has consisted of a large number of consecutive patients, however. Thus, the observations may be thought to represent the characteristics of the general patient population undergoing clinical Holter monitoring examination. Finally, we analyzed only SDNN as the measure of 24-hour HRV in this study, but a variety of indices have been proposed for 24-hour HRV. Their relationships with PA could differ from those of SDNN and need to be investigated for individual indices.

\section{Conclusions}

To clarify the relationship between HRV and PA during daily life, we analyzed the effects of PA in different time zone of the day on SDNN in the ALLSTAR database of ECG and actigraphic data in 51,128 adult patients. 
We found that only daytime PA had a substantial positive effect on SDNN while nighttime PA had a negative effect on it. In adult patients who undergo clinical Holter ECG monitoring, daytime PA is positively associated with increased 24-hour HRV.

\section{References}

[1] Fletcher, G. F., Balady, G., Blair, S. N., Blumenthal, J., Caspersen, C., Chaitman, B., et al. (1996). Statement on exercise: Benefits and recommendations for physical activity programs for all Americans. A statement for health professionals by the Committee on Exercise and Cardiac Rehabilitation of the Council on Clinical Cardiology, American Heart Association. Circulation, 94(4), 857-862.

[2] Thompson, P. D., Buchner, D., Pina, I. L., Balady, G. J., Williams, M. A., Marcus, B. H., et al. (2003). Exercise and physical activity in the prevention and treatment of atherosclerotic cardiovascular disease: A statement from the Council on Clinical Cardiology (Subcommittee on Exercise, Rehabilitation, and Prevention) and the Council on Nutrition, Physical Activity, and Metabolism (Subcommittee on Physical Activity). Circulation, 107(24), 3109-3116

[3] Yamamoto, Y., Hughson, R. L., \& Peterson, J. C. (1991). Autonomic control of heart rate during exercise studied by heart rate variability spectral analysis. J. Appl. Physiol., 71, 1136-1142

[4] Hayano, J., Taylor, J. A., Mukai, S., Okada, A., Watanabe, Y., Takata, K., et al. (1994). Assessment of frequency shifts in R-R interval variability and respiration with complex demodulation. J. Appl. Physiol., 77, 2879-2888

[5] Kleiger, R. E., Miller, J. P., Bigger, J. T., Jr., Moss, A. J., \& the Multicenter Post-Infarction Research, G. (1987). Decreased heart rate variability and its association with increased mortality after acute myocardial infarction. Am. J. Cardiol., 59, 256-262

[6] Watanabe, E., Kiyono, K., Yamamoto, Y., \& Hayano, J. (2016). Heart rate variability and cardiac diseases. Clinical Assessment of the Autonomic Nervous System, 163-178.

[7] Yuda, E., Furukawa, Y., Yoshida, Y., Hayano, J., \& ALLSTAR Research Group. (2016). Association between regional difference in heart rate variability and Inter-prefecture ranking of healthy life expectancy: ALLSTAR big data project in Japan. Proceedings of the 7th EAI International Conference on Big Data Technologies and Applications (BDTA 2016), (p. 6).

[8] Yuda, E., Yoshida, Y., Ogasawara, H., Hayano, J., \& ALLSTAR Research Group. (2016). Ambulatory physical activity monitoring to know healthy life expectancy. Proceedings of the 8th International Workshop on Biosignal Interpretation (BSI2016), USB Paper No.P2-3.

[9] Camm, A. J., Malik, M., Bigger, J. T., Breithardt, G., Cerutti, S., Cohen, R. J., et al. (1996). Task force of the European society of cardiology and the North American Society of pacing and electrophysiology. Heart rate variability: standards of measurement, physiological interpretation and clinical use. Circulation, 93(5), 1043-1065

[10] Billman, G. E. (2009). Cardiac autonomic neural remodeling and susceptibility to sudden cardiac death: Effect of endurance exercise training. Am. J. Physiol. Heart and Circulatory Physiology, 297(4), H1171-1193

[11] Routledge, F. S., Campbell, T. S., McFetridge-Durdle, J. A., \& Bacon, S. L. (2010). Improvements in heart rate variability with exercise therapy. Can. J. Cardiol., 26(6), 303-312.

[12] Hsu, C. Y., Hsieh, P. L., Hsiao, S. F., \& Chien, M. Y. (2015). Effects of exercise training on autonomic function in chronic heart failure: Systematic review. BioMed. Res. Intl., 591708

[13] Buch, A. N., Coote, J. H., \& Townend, J. N. (2002). Mortality, cardiac vagal control and physical training-what's the link? Exp. Physiol., 87(4), 423-435. 


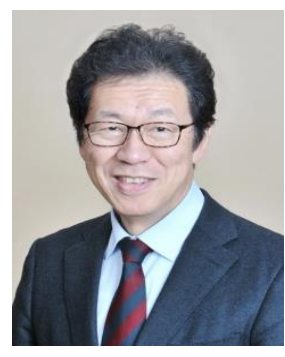

Junichiro Hayano graduated Nagoya City University Medical School, Nagoya, Japan and received the M.D. degree in 1980. From 1981 to 1983, he received residency trainings of psychosomatic medicine in Kyushu University School of Medicine, Fukuoka, Japan. He obtained the Ph.D. degree (Dr. of medical science) in 1988 from Nagoya City University Graduate School of Medical Sciences. From 1990 to 1991, he was working as a visiting associate at the Behavioral Medicine Research Center, Duke University Medical Center, Durham, NC, USA. In 1984, he got a faculty position at Nagoya City University Medical School and has been a Professor of Medicine at Nagoya City University Graduate School of Medical Sciences since 2003. His current interests are applications of dynamic electrocardiography and bio-signal monitoring to cardiology and health sciences.

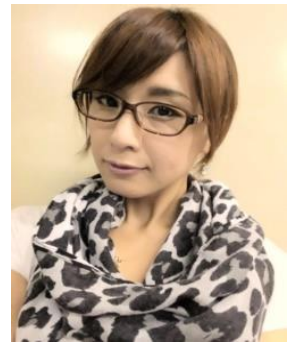

Emi Yuda was born in Mitaka City, Tokyo Japan in 1980. She studied computer science at Moscow University until 2003 and received M.S. degree from Tsukuba University, Japan. She will receive Ph.D. degree from Nihon University graduate school. From 2013 to 2014 she was a research assistant at the Santa Monica College Computer Science department in California, USA. Since 2015, she has been a Project Researcher of New Energy and Industrial Technology Development Organization (NEDO), with the Nagoya City University Graduate School of Medical sciences. Her research is Informatics and Intellectual property. And since 2017 she has been a member of the Steering Committee of the Electronic Intellectual Property Study Group of Information Processing Society of Japan.

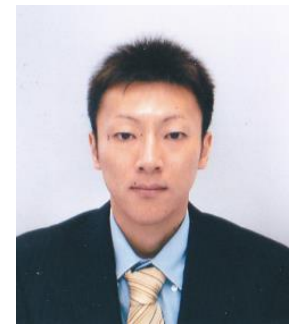

Yutaka Yoshida was born in Aichi, Japan in 1977. He received the B.S. and M.S. degrees in engineering from the Daido University, and Ph.D. degree in information management from Aichi Institute of Technology University, Nagoya, Japan, in 2008. From 2008 to 2012, he was a Research assistant with the Nagoya City University Graduate School of Arts and Sciences. From 2012 to 2016, he was a Project Researcher of "Center of Knowledge", with the Nagoya City University Graduate School of Medical sciences. Since 2016, he has been a Research engineer with the Nagoya City University Graduate School of Medical sciences. His research interests include Bioinformatics engineering and Digital signal processing. 